\title{
Conformal perturbation theory
}

\author{
Andrea Amoretti ${ }^{1, *}$ and Nicodemo Magnoli ${ }^{2,3, \dagger}$ \\ ${ }^{1}$ Physique Théorique et Mathématique Université Libre de Bruxelles, C.P. 231, 1050 Brussels, Belgium \\ ${ }^{2}$ Dipartimento di Fisica, Universita di Genova, Via Dodecaneso 33, 16146 Genova, Italy \\ ${ }^{3}$ INFN, sezione di Genova, Via Dodecaneso 33, 16146 Genova, Italy
}

(Received 16 May 2017; published 29 August 2017)

\begin{abstract}
Statistical systems near a classical critical point have been intensively studied from both theoretical and experimental points of view. In particular, correlation functions are of relevance in comparing theoretical models with the experimental data of real systems. In order to compute physical quantities near a critical point, one needs to know the model at the critical (conformal) point. In this line, recent progress in the knowledge of conformal field theories, through the conformal bootstrap, gives the hope of getting some interesting results also outside of the critical point. In this paper, we will review and clarify how, starting from the knowledge of the critical correlators, one can calculate in a safe way their behavior outside the critical point. The approach illustrated requires the model to be just scale invariant at the critical point. We will clarify the method by applying it to different kind of perturbations of the 2D Ising model.
\end{abstract}

DOI: 10.1103/PhysRevD.96.045016

\section{INTRODUCTION}

Recently, there has been renewed interest in conformal field theories (CFTs) in higher dimensions. Some new insight has been obtained through the conformal bootstrap method [1-5]. One of the most important results has been the computation of the critical exponents and the Wilson coefficients of the 3D Ising model [2,3]. The knowledge of the system at the critical point gives us the possibility to analyze its behavior away from criticality, considering e.g. the perturbations due to the presence of an external magnetic field or an energy operator. The knowledge of the correlators in the vicinity of the critical point is extremely relevant to compare theoretical predictions with experimental data, to study, in the $2 \mathrm{D}$ case, the relation between perturbed CFTs and integrable field theories [6] or the flow between different CFTs [7]. From the theoretical point of view, to obtain the behavior of observables outside a critical point is not an easy task, since usually the standard approach based on the Gell-Mann and Low theorem [8] is not useful in this situation. This is due to the fact that, in the case of a conformal field theory perturbed by a relevant operator, the corrections to the conformal correlation functions are typically plagued by infrared divergences. This problem was first pointed out by Wilson in his seminal paper [9]. In order to understand the issue and its possible solution, it is useful to consider the theory of the free massive boson. It is well known that the Euclidean propagator of this theory can be expressed in terms of a modified Bessel function depending on the product of the mass $m$ and the distance $r$ (times a power of $r$ ). The short distance $(m r \ll 1)$ behavior of the Bessel function contains

\footnotetext{
*andrea.amoretti@ulb.ac.be

†nicodemo.magnoli@ge.infn.it
}

terms proportional to $\log (m r)$, which cannot be obtained by performing a small mass perturbation at any finite order. Additionally, naive perturbative mass expansion is affected by infrared divergences, being the massless "free" theory scale invariant. It is clear that the correlation function by itself has no finite perturbative expansion. As pointed out in Ref. [9], one can overcome the problem by expressing the short distance behavior of the correlators in terms of the operator product expansion (OPE). In this way, the nonperturbative contribution of the correlators, which is encoded in the vacuum expectation values (VEVs) of the local operators in the OPE, separates from the one that can be computed perturbatively, namely the Wilson coefficients. Eventually, if the expectation values of the operator are known, the problem reduces to perturbatively compute the Wilson coefficients.

Even though techniques to perturbatively compute the short distance IR finite behavior of the Wilson coefficients were developed a long time ago [10], it seems that the method is not well known in the recent literature. In this paper, we will review the approach first described in Ref. [10], which can be applied to compute the behavior of the correlators of models of arbitrary space dimensions in the vicinity of a scale invariant critical point. In order to render the method as clear as possible, we will apply it to several examples of perturbations of the 2D Ising model which have not yet been considered in the literature. ${ }^{1}$ Even though the purpose is pedagogical, each example has physical interest by itself, which will be discussed in detail in the conclusions.

\footnotetext{
${ }^{1}$ See e.g. Refs. [11-13] for other examples of perturbations of the 2D Ising model and Ref. [14] for an application to the LeeYang model.
} 
The paper is organized as follows. In Sec. II, we describe the general method to perturbatively compute the Wilson coefficients, clarifying some aspects not discussed in Ref. [10]. In Sec. III, we apply the method to three examples related to the 2D Ising model. First, we consider the correlators of the Ising model perturbed by the energy operator $\mathcal{E}$ in the broken phase, where the spin operator $\sigma$ has a nontrivial expectation value. Specifically, we will compare the perturbative result for the correlator $\langle\sigma \mathcal{E}\rangle$ with the analytical expression obtained by Hecht [15]. This provides us with an example in which conformal perturbation theory gives the correct results even in a phase where the underlying symmetry of the model is somehow broken. Second, we apply the method to the Ising model perturbed by the magnetic field in the presence of a trap. This example might be of relevance to compare theoretical predictions with data from experimental setups in which the presence of a trap is needed to confine the system in a limited region, such as Bose-Einstein condensates and cold atoms [16-19]. Finally, we will show that the method can be used to consistently compute perturbations to the threepoint functions as well. In this case, we will show that the three-point correlation function satisfies an associativity condition outside the critical point that can give nontrivial relations between the Wilson coefficients and its derivatives. The last section of the paper is devoted to conclusions and final remarks.

\section{CONFORMAL PERTURBATION THEORY: A REVIEW}

Let us review the technique derived in Ref. [10], to reconstruct the short distance behavior of the correlators of operators in a D-dimensional Euclidean quantum field theory at a fixed point of the renormalization group, described by the action $S_{e}$, perturbed by one or more relevant operators $\mathcal{O}_{i}$ with couplings $m_{i}$, so that the perturbation to $S_{e}$ takes the form

$$
\Delta S=-\int d^{D} x \sum_{i} m^{i} \mathcal{O}_{i}(x)
$$

Since the operators $\mathcal{O}_{i}$ are supposed to be relevant, their dimensions $x_{i}$ have to satisfy the inequality

$$
0<x_{i}<D \text {. }
$$

In what follows, we will be especially interested in statistical physics applications. In this spirit, the unperturbed theory described by $S_{e}$ is a conformal field theory, and the method which we are going to outline describes a consistent way to go outside the critical point.

In general, the short distance behavior of the correlators of a perturbed field theory is described by the OPE,

$$
\begin{aligned}
& \left\langle\Phi_{a_{1}}\left(r_{1}\right) \ldots \Phi_{a_{n}}\left(r_{n}\right) X\right\rangle_{m} \sim C_{a_{1} \ldots a_{n}}^{c}\left(r_{1}-r_{n}, \ldots, r_{n-1}-r_{n} ; m\right) \\
& \quad \times\left\langle\Phi_{c}\left(r_{n}\right) X(R)\right\rangle_{m},
\end{aligned}
$$

where $\Phi_{i}\left(r_{i}\right)$ describes a complete set of composite operators of dimension $x_{i}, X(R)$ could be either the identity operator $(X(R)=\mathbb{1})$ or a multilocal operator defined on $|R|>|r|, \ldots,\left|r_{n}\right|$, and the index $m$ indicates that the correlators are computed at fixed sources $m_{i}$.

If the unperturbed theory $\left(m_{i}=0\right)$ is a conformal field theory, the convergence of the OPE at $m_{i}=0$ is well understood [20]. However, the convergence of OPE in a general perturbed field theory is far from being proved. By the way, for the sake of the method to be valid, it is sufficient to assume the asymptotic weak convergence of the OPE, namely that the truncated OPE expansion

$$
\begin{aligned}
\Delta_{a_{1} \ldots a_{n}}^{(N)}(X(R), m) \equiv & \left\langle\left(\Phi_{a_{1}}\left(r_{1}\right) \ldots \Phi_{a_{n}}\left(r_{n}\right)\right.\right. \\
& \left.\left.-\sum_{c}^{x_{c} \leq N} C_{a_{1} \ldots a_{n}}^{c}\left(\left\{r_{i}\right\} ; m\right) \Phi_{c}\left(r_{n}\right)\right) X(R)\right\rangle_{m}
\end{aligned}
$$

satisfies the condition

$$
\lim _{N \rightarrow \infty} \lim _{R \rightarrow \infty} \Delta_{a_{1} \ldots a_{n}}^{(N)}(X(R), m) \sim 0
$$

as an asymptotic series in the couplings $m_{i}$. The dependence on the coordinates $\left\{r_{i}\right\}$ of the Wilson coefficients $C_{a_{1} \ldots a_{n}}^{c}$ has to be understood as in (2.3). The condition (2.5) is equivalent to the assumption that arbitrary order derivatives of $\Delta_{a_{1} \ldots a_{n}}^{(N)}$ with respect to the couplings $m_{i}$ asymptotically converge in the $m_{i} \rightarrow 0$ limit, namely

$$
\lim _{N \rightarrow \infty} \lim _{m \rightarrow 0} \lim _{R \rightarrow \infty} \partial_{m_{1}}^{n_{1}} \ldots \partial_{m_{k}}^{n_{k}} \Delta_{a_{1} \ldots a_{n}}^{(N)}(X(R), m)=0
$$

for every $k$ and $n_{i}$.

\section{A. First order expansion}

Having described the basic hypothesis on which we rely, we are now ready to describe the method. For the sake of simplicity, let us first concentrate on the first order expansion. The goal will be to express the first derivative of the Wilson coefficients $C_{a_{1} \ldots a_{n}}^{c}$ with respect to a certain coupling $m_{i}$ in terms of quantities of the unperturbed theory.

From the weak convergence hypothesis (2.6), we know that

$$
\lim _{N \rightarrow \infty} \lim _{m \rightarrow 0} \lim _{R \rightarrow \infty} \partial_{m_{i}} \Delta_{a_{1} \ldots a_{n}}^{(N)}(X(R), m)=0 .
$$

In order to expand the previous expression in a useful way, we notice that, due to the basic action principle [21,22], the following equality holds: 


$$
\partial_{m^{i}}\langle X(R)\rangle_{m}=\int d^{D} x\left\langle: \mathcal{O}_{i}(x): X(R)\right\rangle,
$$

with $\quad: \mathcal{O}_{i}(x): \equiv \mathcal{O}_{i}-\left\langle\mathcal{O}_{i}\right\rangle$.

Keeping in mind the definition of $\Delta_{a_{1} \ldots a_{n}}^{(N)}(X(R), m)$ (2.4) and using (2.8), Eq. (2.7) becomes

$$
\begin{aligned}
\lim _{N \rightarrow \infty} & \lim _{R \rightarrow \infty}\left[\int _ { | \overline { r } | < | R | } d ^ { D } \overline { r } \left\langle: \mathcal{O}_{i}(\bar{r}):\left(\Phi_{a_{1}}\left(r_{1}\right) \ldots \Phi_{a_{n}}\left(r_{n}\right)\right.\right.\right. \\
& \left.\left.-\sum_{c}^{x_{c} \leq N} C_{a_{1} \ldots a_{n}}^{c}\left(\left\{r_{i}\right\}\right) \Phi_{c}\left(r_{n}\right)\right) X(R)\right\rangle \\
& \left.-\sum_{c}^{x_{c} \leq N} \partial_{m_{i}} C_{a_{1} \ldots a_{n}}^{c}\left(\left\{r_{i}\right\}\right)\left\langle\Phi_{c}\left(r_{n}\right) X(R)\right\rangle\right]=0,
\end{aligned}
$$

where the Wilson coefficients $C_{a_{1} \ldots a_{n}}^{c}\left(\left\{r_{i}\right\}\right)$ and the expectation values $\langle\ldots\rangle$ without the index $m$ are the ones evaluated at $m_{i}=0$. The relation (2.9) establishes a set of constraints which involve the first derivative of the Wilson coefficient $C_{a_{1} \ldots a_{n}}^{c}$ with respect to $m_{i}$ and correlators of the unperturbed field theory. Eventually, if one knows the correlators and the Wilson coefficients of the model at the critical point, the system (2.9) can be solved in terms of $\partial_{m_{i}} C_{a_{1} \ldots a_{n}}^{c}$ obtaining the first order $m_{i}$ expansion of the Wilson coefficients.

To further clarify Eq. (2.9), it is worth noting that it can be further simplified if one considers $X(R)=\mathbb{1}$ and noticing that, due to dimensional consideration,

$$
\begin{aligned}
& \lim _{\left|R_{1}\right| \ldots\left|R_{k}\right| \rightarrow \infty} \int_{\left|r_{1}\right|<\left|R_{1}\right|} d^{D} r_{1} \ldots \int_{\left|r_{k}\right|<\left|R_{k}\right|} d^{D} r_{k}\left\langle: \mathcal{O}_{i}\left(r_{1}\right):\right. \\
& \left.\quad \ldots: \mathcal{O}_{k}\left(r_{k}\right): \Phi_{c}\left(r_{n}\right)\right\rangle=0
\end{aligned}
$$

if the dimension $x_{c}$ of $\Phi_{c}$ is less the sum of the mass dimensions $y_{i} \equiv D-x_{i}$ of each operator $\mathcal{O}_{i}$, namely if

$$
x_{c}-\sum_{j=1}^{k} y_{j}>0 \text {. }
$$

This is due to the absence of a physical scale in the unperturbed theory $S_{e}$. With this in mind, the sum in the second term in (2.9) reduces to all the $\Phi_{c}$ with $x_{c}<y_{i}$, while the only nontrivial contribution to the sum in the third term is the one with $\Phi_{c}\left(r_{n}\right)=\mathbb{1}$. Finally, one obtains

$$
\begin{aligned}
\partial_{m_{i}} & C_{a_{1} \ldots a_{n}}^{\mathbb{1}}\left(\left\{r_{i}\right\}\right) \\
= & \lim _{R \rightarrow \infty} \int_{|\bar{r}|<|R|} d^{D} \bar{r}\left\langle: \mathcal{O}_{i}(\bar{r}):\left(\Phi_{a_{1}}\left(r_{1}\right) \ldots \Phi_{a_{n}}\left(r_{n}\right)\right.\right. \\
& \left.\left.-\sum_{c}^{x_{c} \leq y_{i}} C_{a_{1} \ldots a_{n}}^{c}\left(\left\{r_{i}\right\}\right) \Phi_{c}\left(r_{n}\right)\right)\right\rangle .
\end{aligned}
$$

At this point, one might wonder if the previous results might be plagued by ultraviolet divergences. It is worth it to stress that in the $C P T$ of a generic CFT there are no issues concerning ultraviolet divergences, as pointed out in Ref. [10]. On the contrary, the free boson case considered in the Introduction does not belong to this category, and one has to be careful in defining the perturbing composite operator $\phi^{2}$ (see Ref. [10] for a similar discussion in the 2D Ising model and the example III A in this article). In this situation, there appear logarithmic terms in the short distance expansion.

\section{B. Higher order expansion}

In a similar way, one can consider the expansion of the Wilson coefficients up to higher orders in the derivatives with respect to the couplings $m_{i}$. By expanding the relation

$$
\lim _{N \rightarrow \infty} \lim _{R \rightarrow \infty} \lim _{m \rightarrow 0} \partial_{m_{1}} \ldots \partial_{m_{k}} \Delta_{a b}^{(N)}\left(X_{R}, m\right)
$$

and repeating the same steps done in the previous section, one obtains

$$
\begin{aligned}
& \lim _{|R| \rightarrow \infty}\left\{\int_{\left|r_{1}\right|<|R|} d^{D} r_{1} \ldots \int_{\left|r_{k}\right|<|R|} d^{D} r_{k}\left[\left\langle: \mathcal{O}_{i_{k}}: \ldots: \mathcal{O}_{i_{1}}:\left(\Phi_{a_{1}}\left(r_{1}\right) \ldots \Phi_{a_{n}}\left(r_{n}\right)-\sum_{b}^{x_{b} \leq \bar{x}} C_{a_{1} \ldots a_{n}}^{b}\left(\left\{r_{i}\right\}\right) \Phi_{b}\left(r_{n}\right)\right) X(R)\right\rangle\right]\right. \\
& \quad-\sum_{b}^{x_{b} \leq \bar{x}} \partial_{i_{1}} C_{a_{1} \ldots a_{n}}^{b}\left(\left\{r_{i}\right\}\right) \int_{\left|r_{2}\right|<|R|} d^{d} r_{2} \ldots \int_{\left|r_{k}\right|<|R|} d^{D} r_{k}\left\langle: \mathcal{O}_{i_{k}}: \ldots: \mathcal{O}_{i_{2}}: \Phi_{b}\left(r_{n}\right) X(R)\right\rangle \\
& \quad \ldots \\
& \left.\quad-\sum_{b}^{x_{b} \leq \bar{x}} \partial_{i_{1}} \ldots \partial_{i_{k-1}} C_{a_{1} \ldots a_{n}}^{b}\left(\left\{r_{i}\right\}\right) \int_{\left|r_{k}\right|<|R|}\left\langle: \mathcal{O}_{i_{k}}: \Phi_{b}\left(r_{n}\right) X(R)\right\rangle-\sum_{b}^{x_{b} \leq \bar{x}} \partial_{i_{1}} \ldots \partial_{i_{k}} C_{a_{1} \ldots a_{n}}^{b}\left(\left\{r_{i}\right\}\right)\left\langle\Phi_{b}\left(r_{n}\right) X(R)\right\rangle\right\},
\end{aligned}
$$


where $\bar{x}=\sum_{k} y_{i_{k}}-x_{X(R)}$. For generic $X(R)$, the previous relations provide a set of constraints which relate the derivatives of the Wilson coefficients with respect to the coupling to correlators of the unperturbed theory. This system can be consistently solved in terms of the derivatives of the Wilson coefficients if one knows the properties of the model at the critical point.

Once again, we can simplify the previous relation considering $X(R)=\mathbb{1}$, finally obtaining an equality for the derivatives of $C_{a_{1} \ldots a_{n}}^{\mathbb{1}}$, namely

$$
\begin{aligned}
\partial_{i_{1}} \ldots \partial_{i_{k}} C_{a_{1} \ldots a_{n}}^{\|}\left(\left\{r_{i}\right\}\right) & =\lim _{|R| \rightarrow \infty}\left\{\int_{\left|r_{1}\right|<|R|} d^{D} r_{1} \ldots \int_{\left|r_{k}\right|<|R|} d^{D} r_{k}\left[\left\langle: \mathcal{O}_{i_{k}}: \ldots: \mathcal{O}_{i_{1}}:\left(\Phi_{a_{1}}\left(r_{1}\right) \ldots \Phi_{a_{n}}\left(r_{n}\right)-\sum_{b}^{x_{b} \leq \bar{x}} C_{a_{1} \ldots a_{n}}^{b}\left(\left\{r_{i}\right\}\right) \Phi_{b}\left(r_{n}\right)\right)\right\rangle\right]\right. \\
& -\sum_{b}^{x_{b} \leq \bar{x}} \partial_{i_{1}} C_{a_{1} \ldots a_{n}}^{b}\left(\left\{r_{i}\right\}\right) \int_{\left|r_{2}\right|<|R|} d^{d} r_{2} \ldots \int_{\left|r_{k}\right|<|R|} d^{D} r_{k}\left\langle: \mathcal{O}_{i_{k}}: \ldots: \mathcal{O}_{i_{2}}: \Phi_{b}\left(r_{n}\right)\right\rangle \\
& \ldots \\
& \left.-\sum_{b}^{x_{b} \leq \bar{x}} \partial_{i_{1}} \ldots \partial_{i_{k-1}} C_{a_{1} \ldots a_{n}}^{b}\left(\left\{r_{i}\right\}\right) \int_{\left|r_{k}\right|<|R|}\left\langle: \mathcal{O}_{i_{k}}: \Phi_{b}\left(r_{n}\right)\right\rangle\right\} .
\end{aligned}
$$

As a final comment, we note that, having obtained the relations (2.9) and (2.14), one has to prove that the expansion is IR finite, namely that all the nonperturbative contributions of the correlators are encoded in the expectations values of the operators in the OPE (2.3). The proof has been outlined in Ref. [10], and since it goes beyond the purposes of the present paper, we refer to Ref. [10] for further details.

\section{EXAMPLES: 2D ISING MODEL APPLICATIONS}

In this section, we will describe some applications of the conformal perturbation theory techniques previously described to the 2D Ising model. As known (see e.g. Ref. [23]), at the critical point, $T=T_{c}$, the Ising model is described by the continuous unitary conformal field theory $\mathcal{M}(3 / 4)$. The primary operators of this conformal field theory are $\mathbb{1}, \sigma$, and $\mathcal{E}$, with dimensions $x=0,1 / 8$, and 1 respectively. The corresponding fusion rules are

$[\sigma][\sigma]=[\mathbb{1}]+[\mathcal{E}], \quad[\mathcal{E}][\mathcal{E}]=\mathbb{1}, \quad[\sigma][\mathcal{E}]=[\sigma]$.

The previous relations imply that correlation functions involving an odd number of $\sigma \mathrm{s}$ identically vanish.

In order to set the conventions and the normalization, we list below some of the correlation functions and Wilson coefficients of this theory which will be useful for our purposes in the following sections,

$$
\begin{array}{ll}
C_{\mathcal{E} \mathcal{Q}}^{\mathbb{1}}(z)=\frac{1}{|z|^{2}}, & C_{\sigma \sigma}^{\mathbb{1}}(z)=\frac{1}{|z|^{\frac{1}{4}}}, \\
C_{\sigma \sigma}^{\mathcal{E}}(z)=\frac{|z|^{\frac{3}{4}}}{2}, & C_{\sigma \mathcal{E}}^{\sigma}(z)=\frac{1}{2|z|},
\end{array}
$$

and

$$
\begin{gathered}
\left\langle\sigma\left(z_{1}\right) \sigma\left(z_{2}\right) \mathcal{E}\left(z_{3}\right)\right\rangle=\frac{\left|z_{12}\right|^{\frac{3}{4}}}{2\left|z_{13}\right|\left|z_{23}\right|}, \\
\left\langle\sigma\left(z_{1}\right) \sigma\left(z_{2}\right) \mathcal{E}\left(z_{3}\right) \mathcal{E}\left(z_{4}\right)\right\rangle=\frac{\left|z_{12}\left(z_{32}+z_{42}\right)-2 z_{32} z_{42}\right|^{2}}{4\left|z_{42} z_{32} z_{41} z_{31}\right|\left|z_{43}\right|^{2}\left|z_{12}\right|^{\frac{1}{4}}},
\end{gathered}
$$

$$
\begin{aligned}
& \left\langle\sigma\left(z_{1}\right) \sigma\left(z_{2}\right) \sigma\left(z_{3}\right) \sigma\left(z_{4}\right)\right\rangle \\
& \quad=\left|(1-x) z_{12} z_{34}\right|^{-\frac{1}{4}}\left(\left|\frac{1+\sqrt{1-x}}{2}\right|+\left|\frac{1-\sqrt{1-x}}{2}\right|\right),
\end{aligned}
$$

where $z$ is a complex coordinate in the $2 D$ plane, $z_{i j} \equiv z_{i}-z_{j}$, and $x=\frac{z_{12} z_{34}}{z_{13} z_{24}}$.

\section{A. Thermally perturbed two-point functions}

For a first example, we consider a case in which we move away from the critical point by perturbing the Ising model with the energy operator, namely

$$
S=S_{\text {Ising }}+\lambda \int \mathcal{E}(z) d^{2} z
$$

Let us analyze the two-point function $\left\langle\mathcal{E}\left(z_{1}\right) \sigma\left(z_{2}\right)\right\rangle_{\lambda}$. Assuming the operator product expansion is valid outside the critical point, we obtain

$$
\begin{aligned}
\left\langle\mathcal{E}\left(z_{1}\right) \sigma\left(z_{2}\right)\right\rangle_{\lambda}= & C_{\mathcal{E} \sigma}^{\mathbb{1}}\left(z_{12} ; \lambda\right)+C_{\mathcal{E} \sigma}^{\mathcal{E}}\left(z_{12} ; \lambda\right)\langle\mathcal{E}\rangle_{\lambda} \\
& +C_{\mathcal{E} \sigma}^{\sigma}\left(z_{12} ; \lambda\right)\langle\sigma\rangle_{\lambda} .
\end{aligned}
$$


The previous correlator vanishes identically at $\lambda=0$ since it contains just one insertion of $\sigma$. Moreover, by using the relation (2.15), one can easily prove that $C_{\mathcal{E} \sigma}^{\mathbb{1}}\left(z_{12} ; \lambda\right)$ and $C_{\mathcal{E} \sigma}^{\mathcal{E}}\left(z_{12} ; \lambda\right)$ vanishes identically at all orders in perturbation theory, since the correlation functions that one has to compute always contain an odd number of insertions of $\sigma$. Eventually, we find that, outside the critical point, the correlator is proportional to the expectation value of $\sigma$, namely

$$
\left\langle\mathcal{E}\left(z_{1}\right) \sigma\left(z_{2}\right)\right\rangle_{\lambda}=C_{\mathcal{E} \sigma}^{\sigma}\left(z_{12} ; \lambda\right)\langle\sigma\rangle_{\lambda},
$$

and is nonzero only in the magnetically ordered phase. Expanding (3.8) up to the first order in the coupling $\lambda$, we find

$$
\left\langle\mathcal{E}\left(z_{1}\right) \sigma\left(z_{2}\right)\right\rangle_{\lambda}=\left(C_{\mathcal{E} \sigma}^{\sigma}\left(z_{12}\right)+\lambda \partial_{\lambda} C_{\mathcal{E} \sigma}^{\sigma}\left(z_{12}\right)+\cdots\right)\langle\sigma\rangle_{\lambda},
$$

where the dots stand for higher order corrections. Regarding the expectation value of $\sigma$, by dimensional analysis, we obtain

$$
\langle\sigma\rangle_{\lambda}=A_{\sigma} \lambda^{\frac{1}{8}}
$$

where $A_{\sigma}$ is a nonuniversal constant. To compute $C_{\mathcal{E} \sigma}^{\sigma}\left(z_{12}\right)$, we rely on the operator product expansion (2.3) and on the orthogonality of the two-point correlation functions at the critical point, namely

$$
\begin{aligned}
C_{\mathcal{E} \sigma}^{\sigma}\left(z_{12}\right) & =\lim _{\left|z_{3}\right| \rightarrow \infty} \frac{\left\langle\sigma\left(z_{1}\right) \sigma\left(z_{3}\right) \mathcal{E}\left(z_{2}\right)\right\rangle}{\left\langle\sigma\left(z_{1}\right) \sigma\left(z_{3}\right)\right\rangle} \\
& =\lim _{\left|z_{3}\right| \rightarrow \infty} \frac{\left|z_{13}\right|}{2\left|z_{12}\right|\left|z_{23}\right|}=\frac{1}{2\left|z_{12}\right|} .
\end{aligned}
$$

In order to evaluate the second term of the parentheses in (3.9), we use the relation (2.9) derived in the previous section. Specifically, by setting $X=\sigma$, we obtain

$$
\begin{aligned}
\partial_{\lambda} & C_{\mathcal{E} \sigma}^{\sigma}\left(z_{12}\right) \lim _{\left|z_{2}\right| \rightarrow \infty}\left\langle\sigma\left(z_{2}\right) \sigma\left(z_{4}\right)\right\rangle \\
= & \lim _{\left|z_{4}\right| \rightarrow \infty} \int_{\left|z_{3}\right|<\left|z_{4}\right|} d^{2} z_{3}\left[\left\langle\sigma\left(z_{2}\right) \sigma\left(z_{4}\right) \mathcal{E}\left(z_{1}\right) \mathcal{E}\left(z_{3}\right)\right\rangle\right. \\
& -C_{\sigma \mathcal{E}}^{\sigma}\left(z_{12}\right)\left\langle\sigma\left(z_{2}\right) \sigma\left(z_{4}\right) \mathcal{E}\left(z_{3}\right)\right\rangle \\
& \left.-C_{\sigma \mathcal{E}}^{\sigma_{1}}\left(z_{12}\right)\left\langle\sigma_{1}\left(z_{2}\right) \sigma\left(z_{4}\right) \mathcal{E}\left(z_{3}\right)\right\rangle\right],
\end{aligned}
$$

where $\sigma_{1} \equiv L_{-1} \bar{L}_{-1} \sigma$ and $L_{n}$ are the Virasoro operators.

The last term in (3.12) does not contribute to the integral. Regarding the other two terms, after dividing for $\left\langle\sigma\left(z_{2}\right) \sigma\left(z_{4}\right)\right\rangle$, performing the $\left|z_{4}\right| \rightarrow \infty$ limit, and setting $z_{1}=0$ and $z_{2}=z^{2}$, we obtain

\footnotetext{
${ }^{2}$ This can be done due to conformal invariance at the critical point.
}

$$
\partial_{\lambda} C_{\mathcal{E} \sigma}^{\sigma}(z)=\int d^{2} z_{3} \frac{z_{3} \bar{z}+\bar{z}_{3} z}{2\left|z_{3}\right||z|\left|z-z_{3}\right|^{2}} .
$$

The integral has an UV divergence when $z_{3}$ approaches $z$. We regularize it by setting a cutoff at $\left|z_{3}\right|=|z|+\epsilon$. The final result is

$$
\partial_{\lambda} C_{\mathcal{E} \sigma}^{\sigma}(z)=2 \pi\left(\log \frac{2|z|}{\epsilon}-1\right)
$$

To get rid of the UV regulator, one has to consider the connected part of the correlator by subtracting from (3.9) the quantity $\left\langle\mathcal{E}\left(z_{1}\right)\right\rangle_{\lambda}\left\langle\sigma\left(z_{2}\right)\right\rangle_{\lambda}$. In order to do this, we need to compute the expectation value $\left\langle\mathcal{E}\left(z_{1}\right)\right\rangle_{\lambda}$. This has been done in Ref. [10] by noting that the action principle has to be valid, namely

$\partial_{\lambda}\langle\mathcal{E}(0)\rangle_{\lambda}=\int d^{2} z_{2}\left\langle\mathcal{E}\left(z_{2}\right) \mathcal{E}(0)\right\rangle_{\lambda}=-2 \pi \lambda \log 2 \pi \lambda e^{\gamma_{E}} \epsilon$,

where we refer to Ref. [10] for the details of the computation. Putting all together, we find

$$
\begin{aligned}
& \left\langle\mathcal{E}\left(z_{1}\right) \sigma\left(z_{2}\right)\right\rangle_{\lambda}-\left\langle\mathcal{E}\left(z_{1}\right)\right\rangle_{\lambda}\left\langle\sigma\left(z_{2}\right)\right\rangle_{\lambda} \\
& \quad=A_{\sigma} \lambda^{\frac{1}{8}}\left(\frac{1}{2\left|z_{12}\right|}+2 \pi \lambda\left(\log 4 \pi \lambda\left|z_{12}\right|-1+\gamma_{E}\right)+\cdots\right) .
\end{aligned}
$$

Note that the computation (3.15) is nonperturbative, since it, as explained in Ref. [10], relies on the exact $\langle\mathcal{E E}\rangle_{\lambda}$, which is known analytically outside the critical point for the special case of the 2D Ising model. As a consequence, it is important to verify, as we have done, that the connected Green's function (3.16) does not depend on the UV regulator $\epsilon$. The expansion (3.16) is in perfect agreement with the exact result of the correlator found in Ref. [15]:

$$
\begin{gathered}
\left\langle\mathcal{E}\left(z_{1}\right) \sigma\left(z_{2}\right)\right\rangle_{\lambda}-\left\langle\mathcal{E}\left(z_{1}\right)\right\rangle_{\lambda}\left\langle\sigma\left(z_{2}\right)\right\rangle_{\lambda} \\
=2 \pi \lambda A_{\sigma} \lambda^{\frac{1}{8}} \int_{4 \pi \lambda\left|z_{12}\right|}^{\infty} d s s^{-2} e^{-s} .
\end{gathered}
$$

\section{B. Trapped Ising model}

For a second example, inspired by the analysis of Ref. [24], we consider the 2D Ising model with a trap perturbation, namely

$$
\begin{aligned}
S & =S_{\mathrm{CFT}}+\int d^{2} z U(z) \sigma(z), \quad \text { with } \\
U(z) & =v^{p}|z|^{p} \equiv \rho|z|^{p} .
\end{aligned}
$$


In the previous expression, $p(\geq 2)$ is a generic exponent of the trap potential, while $\rho$ is the characteristic trap parameter. In what follows, we will consider the effect of a small $\rho$ perturbation, namely the limit of a large trap, on the two-point functions of the Ising model. As explained in Ref. [24], this might be of relevance in experimental studies of trapped critical systems such as cold atoms and Bose-Einstein condensates.

As noted in Refs. [24,25], by using renormalization group arguments, one can deduce the scaling behavior of the expectation value of the spin and the energy operator in the center of the trap,

$$
\langle\sigma(0)\rangle_{\rho}=B_{\sigma} \rho^{\frac{\theta}{8}}, \quad\langle\mathcal{E}(0)\rangle_{\rho}=B_{\mathcal{E}} \rho^{\theta},
$$

where the exponent $\theta=\frac{8}{15+8 p}$ is the characteristic trap exponent, while $B_{\sigma}$ and $B_{\mathcal{E}}$ are nonuniversal constants.

The perturbation that we are considering breaks translational invariance but preserves rotational symmetry. Hence, the fusion rule of the perturbed model remains the same as the unperturbed one, and if we fix one operator in the center of the trap, it is possible to rely on the OPE and on the perturbative techniques described in the previous sections to compute the two-point functions outside the critical point. The previous argument yields

$$
\begin{aligned}
& \left\langle\sigma\left(z_{1}\right) \sigma(0)\right\rangle_{\rho}=C_{\sigma \sigma}^{\rrbracket}\left(z_{1}\right)+C_{\sigma \sigma}^{\mathcal{E}}\left(z_{1}\right) B_{\mathcal{E}} \rho^{\theta} \\
& +\partial_{\rho} C_{\sigma \sigma}^{\sigma}\left(z_{1}\right) B_{\sigma} \rho^{\frac{\theta}{8}+1}+\cdots, \\
& \left\langle\mathcal{E}\left(z_{1}\right) \mathcal{E}(0)\right\rangle_{\rho}=C_{\mathcal{E} \mathcal{E}}^{\Uparrow}\left(z_{1}\right)+\partial_{\rho} C_{\mathcal{E} \mathcal{E}}^{\sigma}\left(z_{1}\right) B_{\sigma} \rho^{\frac{\theta}{8}+1}+\cdots, \\
& \left\langle\sigma\left(z_{1}\right) \mathcal{E}(0)\right\rangle_{\rho}=C_{\sigma \mathcal{E}}^{\sigma}\left(z_{1}\right) B_{\sigma} \rho^{\frac{\theta}{8}}+\rho \partial_{\rho} C_{\sigma \mathcal{E}}^{\mathbb{Q}}\left(z_{1}\right) \\
& +\partial_{\rho} C_{\sigma \mathcal{E}}^{\mathcal{E}}\left(z_{1}\right) B_{\mathcal{E}} \rho^{\theta+1} \ldots
\end{aligned}
$$

The last terms in (3.20), (3.21), and (3.22) can be computed by using the conformal perturbation theory techniques described in the previous sections. Specifically, one has to evaluate the following integrals:

$$
\begin{aligned}
& -\partial_{\rho} C_{\sigma \sigma}^{\sigma}\left(z_{1}\right) \lim _{\left|z_{3}\right| \rightarrow \infty}\left\langle\sigma\left(z_{3}\right) \sigma(0)\right\rangle \\
& =\lim _{\left|z_{3}\right| \rightarrow \infty} \int_{\left|z_{2}\right|<\left|z_{3}\right|} d^{2} z_{2}\left|z_{2}\right|^{p}\left[\left\langle\sigma\left(z_{1}\right) \sigma\left(z_{2}\right) \sigma\left(z_{3}\right) \sigma(0)\right\rangle\right. \\
& \left.\quad-C_{\sigma \sigma}^{\mathbb{1}}\left(z_{1}\right)\left\langle\sigma\left(z_{2}\right) \sigma\left(z_{3}\right)\right\rangle-C_{\sigma \sigma}^{\mathcal{E}}\left(z_{1}\right)\left\langle\sigma\left(z_{2}\right) \sigma\left(z_{3}\right) \mathcal{E}(0)\right\rangle\right],
\end{aligned}
$$

$$
\begin{aligned}
- & \partial_{\rho} C_{\mathcal{E E}}^{\sigma}\left(z_{1}\right) \lim _{\left|z_{3}\right| \rightarrow \infty}\left\langle\sigma\left(z_{3}\right) \sigma(0)\right\rangle \\
= & \lim _{\left|z_{3}\right| \rightarrow \infty} \int_{\left|z_{2}\right|<\left|z_{3}\right|} d^{2} z_{2}\left|z_{2}\right|^{p}\left[\left\langle\sigma\left(z_{2}\right) \sigma\left(z_{3}\right) \mathcal{E}\left(z_{1}\right) \mathcal{E}(0)\right\rangle\right. \\
& \left.-C_{\mathcal{E E}}^{\mathbb{1}}\left(z_{1}\right)\left\langle\sigma\left(z_{2}\right) \sigma\left(z_{3}\right)\right\rangle\right],
\end{aligned}
$$

$$
\begin{aligned}
- & \partial_{\rho} C_{\sigma \mathcal{E}}^{\mathbb{E}}\left(z_{1}\right)= \\
- & \int d^{2} z_{2}\left|z_{2}\right|^{p}\left[\left\langle\sigma\left(z_{1}\right) \sigma\left(z_{2}\right) \mathcal{E}(0)\right\rangle\right. \\
& \left.-C_{\sigma \mathcal{E}}^{\sigma}\left(z_{1}\right)\left\langle\sigma\left(z_{2}\right) \sigma(0)\right\rangle\right], \\
- & \partial_{\rho} C_{\sigma \mathcal{E}}^{\mathcal{E}}\left(z_{1}\right) \lim _{\left|z_{3}\right| \rightarrow \infty}\left\langle\mathcal{E}\left(z_{3}\right) \mathcal{E}(0)\right\rangle \\
= & \lim _{\left|z_{3}\right| \rightarrow \infty} \int_{\left|z_{2}\right|<\left|z_{3}\right|} d^{2} z_{2}\left|z_{2}\right|^{p}\left[\left\langle\sigma\left(z_{1}\right) \sigma\left(z_{2}\right) \mathcal{E}\left(z_{3}\right) \mathcal{E}(0)\right\rangle\right. \\
& -C_{\sigma \mathcal{E}}^{\sigma}\left(z_{1}\right)\left\langle\sigma\left(z_{2}\right) \mathcal{E}\left(z_{3}\right) \sigma(0)\right\rangle \\
& \left.-C_{\sigma \mathcal{E}}^{\sigma^{1}}\left(z_{1}\right)\left\langle\sigma\left(z_{2}\right) \mathcal{E}\left(z_{3}\right) \sigma^{1}(0)\right\rangle\right] .
\end{aligned}
$$

A detailed discussion on how to treat the last expressions can be found in the Appendix A. Here, we outline the results in the case of the harmonic trap $(p=2)$, which is the relevant one in typical experimental setups [24]:

$$
\begin{gathered}
\partial_{\rho} C_{\mathcal{E} \mathcal{E}}^{\sigma}\left(z_{1}, p=2\right)=\frac{\left|z_{1}\right|^{2}}{64} \pi\left(3+2 \gamma_{E}-2 \log (4)\right) \\
\partial_{\rho} C_{\sigma \mathcal{E}}^{1}\left(z_{1}, p=2\right)=-\left|z_{1}\right|^{\frac{11}{4}} \frac{\pi \cot \left(\frac{\pi}{8}\right) \Gamma\left(\frac{11}{8}\right)^{2}}{8 \Gamma\left(\frac{23}{8}\right)^{2}} \\
\partial_{\rho} C_{\sigma \mathcal{E}}^{\mathcal{E}}\left(z_{1}, p=2\right)=-\left|z_{1}\right|^{\frac{15}{4}} \frac{961 \Gamma\left(-\frac{19}{8}\right)^{2} \Gamma\left(\frac{7}{8}\right)^{2}}{2048 \sqrt{2} \pi} \\
\partial_{\rho} C_{\sigma \sigma}^{\sigma}\left(z_{1}, p=2\right)=-\left|z_{1}\right|^{\frac{15}{4}} 0.00153398 .
\end{gathered}
$$

In order to make contact with the experiments, it is useful to express the final result in a Fourier transform. In order to do this, one has to keep in mind that for $n \neq 2 \mathbb{Z}$ (see e.g. Ref. [26])

$$
\mathcal{F}\left[|z|^{n}\right](|q|)=\frac{2^{n} n \Gamma\left(\frac{n}{2}\right)|q|^{-n-2}}{\Gamma\left(-\frac{n}{2}\right)},
$$

while

$$
\begin{aligned}
& \mathcal{F}\left[|z|^{2}\right](q, \bar{q})=-2 \pi \partial_{q} \partial_{\bar{q}} \delta(q) \delta(\bar{q}), \\
& \mathcal{F}\left[|z|^{-2}\right](|q|)=-\log \frac{|q|}{2 \mu}-\gamma_{E},
\end{aligned}
$$

where $\mu$ is a renormalization parameter. The last equivalence in (3.32) has been obtained by expanding (3.31) around the singular point $n=-2$ and taking the finite part.

Eventually, the Fourier transform of the correlators (3.20)-(3.22) is

$$
\begin{aligned}
\langle\sigma(q) \sigma(-q)\rangle_{\rho}= & \frac{1}{|q|^{23 / 4}}\left(-0.3908 B_{\mathcal{E}} \rho^{\theta}|q|^{3}\right. \\
& \left.-0.01607 B_{\sigma} \rho^{\theta / 8+1}+0.2432|q|^{4}\right)
\end{aligned}
$$




$$
\begin{aligned}
\langle\mathcal{E}(q) \mathcal{E}(-q)\rangle_{\rho}= & -\log \frac{|q|}{2 \mu}-\gamma_{E} \\
& -0.42620 B_{\sigma} \rho^{\frac{\theta}{8}+1} \partial_{q} \partial_{\bar{q}} \delta(q) \delta(\bar{q}) \\
\langle\sigma(q) \mathcal{E}(-q)\rangle_{\rho}= & -\frac{1.8026}{q^{23 / 4}} B_{\mathcal{E}} \rho^{\theta+1}+\frac{B_{\sigma} \rho^{\theta / 8}}{q}-\frac{1.38552}{q^{19 / 4}} \rho .
\end{aligned}
$$

\section{Magnetically perturbed three-point functions}

As we pointed out in the first section, $C P T$ can be also applied to three-point correlators. ${ }^{3}$ In what follows, we will analyze the 2D Ising model perturbed by a magnetic field $h$, namely

$$
S=S_{\text {Ising }}+h \int \sigma(z) d^{2} z
$$

We will apply the conformal perturbation theory techniques described in Sec. II to the study of three-point correlation functions outside the critical point. Specifically, we will analyze $\left\langle\sigma\left(z_{1}\right) \sigma\left(z_{2}\right) \mathcal{E}\left(z_{3}\right)\right\rangle_{h}$. The assumption that the OPE is valid outside the critical point implies

$$
\begin{aligned}
\left\langle\sigma\left(z_{1}\right) \sigma\left(z_{2}\right) \mathcal{E}\left(z_{3}\right)\right\rangle_{h}= & C_{\sigma \sigma \mathcal{E}}^{1}\left(z_{1}, z_{2}, z_{3} ; h\right) \\
& +C_{\sigma \sigma \mathcal{E}}^{\sigma}\left(z_{1}, z_{2}, z_{3} ; h\right)\langle\sigma\rangle_{h} \\
& +C_{\sigma \sigma \mathcal{E}}^{\mathcal{E}}\left(z_{1}, z_{2}, z_{3} ; h\right)\langle\mathcal{E}\rangle_{h} .
\end{aligned}
$$

The leading correction to the correlation function comes from the term proportional to $\langle\mathcal{E}\rangle_{h}$, namely

$$
\begin{aligned}
\left\langle\sigma\left(z_{1}\right) \sigma\left(z_{2}\right) \mathcal{E}\left(z_{3}\right)\right\rangle_{h}= & C_{\sigma \sigma \mathcal{E}}^{\mathbb{1}}\left(z_{1}, z_{2}, z_{3}\right) \\
& +C_{\sigma \sigma \mathcal{E}}^{\mathcal{E}}\left(z_{1}, z_{2}, z_{3}\right)\langle\mathcal{E}\rangle_{h}+\cdots .
\end{aligned}
$$

In order to evaluate the previous expression, we need the quantities $C_{\sigma \sigma \mathcal{E}}^{\mathbb{Q}}\left(z_{1}, z_{2}, z_{3}\right)$ and $C_{\sigma \sigma \mathcal{E}}^{\mathcal{E}}\left(z_{1}, z_{2}, z_{3}\right)$, as well as the expectation value $\langle\mathcal{E}\rangle_{h}$. Dimensional analysis tells us that the latter one is given by

$$
\langle\mathcal{E}\rangle_{h}=A_{\mathcal{E}} h^{\frac{8}{15}},
$$

where $A_{\mathcal{E}}$ is a nonuniversal constant. Moreover, by definition, $C_{\sigma \sigma \mathcal{E}}^{\mathbb{1}}\left(z_{1}, z_{2}, z_{3}\right)$ is given by

$C_{\sigma \sigma \mathcal{E}}^{\mathbb{1}}\left(z_{1}, z_{2}, z_{3}\right)=\left\langle\sigma\left(z_{1}\right) \sigma\left(z_{2}\right) \mathcal{E}\left(z_{3}\right)\right\rangle=\frac{\left|z_{12}\right|^{\frac{3}{4}}}{2\left|z_{13}\right|\left|z_{23}\right|}$,

where we have used (3.4). Finally, in order to compute $C_{\sigma \sigma \mathcal{E}}^{\mathcal{E}}\left(z_{1}, z_{2}, z_{3}\right)$, we rely, as in the previous subsection, on

\footnotetext{
${ }^{3}$ See Ref. [27] for an example using the Potts model.
}

the operator product expansion (2.3) together with the orthogonality of the two-point functions at the critical point. Eventually, in the limit $\left|z_{4}\right| \rightarrow \infty$, and using the Eqs. (3.2) and (3.4), we obtain

$$
\begin{aligned}
C_{\sigma \sigma \mathcal{E}}^{\mathcal{E}}\left(z_{1}, z_{2}, z_{3}\right) & =\lim _{\left|z_{4}\right| \rightarrow \infty} \frac{\left\langle\sigma\left(z_{1}\right) \sigma\left(z_{2}\right) \mathcal{E}\left(z_{3}\right) \mathcal{E}\left(z_{4}\right)\right\rangle}{\left\langle\mathcal{E}\left(z_{3}\right) \mathcal{E}\left(z_{4}\right)\right\rangle} \\
& =\lim _{\left|z_{4}\right| \rightarrow \infty} \frac{\left|z_{12}\left(z_{32}+z_{42}\right)-2 z_{32} z_{42}\right|^{2}}{4\left|z_{42} z_{32} z_{41} z_{31}\right|\left|z_{12}\right|^{\frac{1}{4}}} .
\end{aligned}
$$

Performing the $\left|z_{4}\right| \rightarrow \infty$ limit, we get

$$
C_{\sigma \sigma \mathcal{E}}^{\mathcal{E}}\left(z_{1}, z_{2}, z_{3}\right)=\frac{1}{4} \frac{\left|z_{12}-2 z_{32}\right|^{2}}{\left|z_{32} z_{31}\right|\left|z_{12}\right|^{\frac{1}{4}}} .
$$

Putting all together, we finally obtain

$$
\begin{aligned}
& \left\langle\sigma\left(z_{1}\right) \sigma\left(z_{2}\right) \mathcal{E}\left(z_{3}\right)\right\rangle_{h} \\
& \quad=\frac{1}{2} \frac{\left|z_{12}\right|^{\frac{3}{4}}}{\left|z_{13} z_{23}\right|}\left(1+A_{\mathcal{E}} h^{8 / 15} \frac{\left|z_{13}+z_{23}\right|^{2}}{2\left|z_{12}\right|}+\cdots\right),
\end{aligned}
$$

where the dots stand for higher order correction in the magnetic field $h$. Finally, we note that in the limit $\left|z_{3}\right| \gg$ $\left|z_{1}\right|,\left|z_{2}\right| \gg\left|z_{1}\right|$ the following operator product expansion relation holds:

$$
\left\langle\sigma\left(z_{1}\right) \sigma\left(z_{2}\right) \mathcal{E}\left(z_{3}\right)\right\rangle_{h}=\left\langle\sigma\left(z_{1}\right) \sigma\left(z_{2}\right)\right\rangle_{h}\left\langle\mathcal{E}\left(z_{3}\right)\right\rangle_{h}=\frac{A_{\mathcal{E}} h^{\frac{8}{15}}}{\left|z_{12}\right|^{\frac{1}{4}}} .
$$

\section{CONCLUSIONS}

In this paper, we have shown that in order to get infrared finite correlation functions near a critical point one has to modify the traditional approach to perturbation theory. We have illustrated the correct approach by giving various nontrivial examples of its application. Specifically, we have applied the method to compute the corrections due to several kinds of relevant perturbations of the 2D Ising model not yet considered in the literature. In the special 2D case, the computation of the VEVs can be performed, relying only on the knowledge of the CFT model using the truncated conformal space approach [28], and this simplifies the discussion. However, it is worth mentioning that the method illustrated in Sec. II is valid in any space-time dimension, and it has already been applied to the 3D Ising model [29-31], even though, at present, in the 3D case the computations of VEVs rely only on numerical simulations.

For a first example, we have considered the perturbation due to the energy operator. In particular, we have proven that, in this case, the conformal perturbation theory result for the correlator $\langle\sigma \mathcal{E}\rangle$ totally agrees with the exact correlator in the broken phase obtained in Ref. [15]. This might be seen 
as a first step in the direction of applying the method to higher-dimensional models which exhibit a spontaneous symmetry breaking, as the 3D $O(N)$ model, where recent conformal bootstrap computations of the Wilson coefficient at the critical point [32] may render the method described here applicable. In this case, the dynamics of Goldstone bosons might be relevant, and recently, it was pointed out in Ref. [33] that they might be the responsible for $C P T$ to not reproduce correctly logarithmic terms in the current-current correlator. The method that we have illustrated just relies on the validity of the OPE outside the critical point. Since, as observed in Ref. [34], the OPE should also be valid in case of spontaneous symmetry breaking, it is worth it to analyze if it is possible, by applying this method, to reproduce the correct result for the current-current correlator in the $O(N)$ model. $^{4}$

For a second example, we have considered the perturbation due to the presence of a trap. This example might be of relevance from both the experimental and theoretical points of view. From the experimental side, the present analysis might be useful for comparing the results with experimental data on trapped systems such as cold atoms [16-19]. From the theoretical point of view, it is worth it to mention that the trap case has some similarities with considering the system at finite temperature. In fact, the temperature acts as a box in the Euclidean time direction, so that the VEV will be modified, but the Wilson coefficients will remain unchanged. In this direction, the method could be applied to compute the response functions near a quantum critical point at zero or finite temperature [33].

With the third example, we have proven that the method can be extremely powerful also in computing correction to the $n$ th-point correlation functions. In particular, we have analyzed the corrections to the three-point function $\langle\sigma \sigma \mathcal{E}\rangle$ due to the presence of an external magnetic field. As a possible future direction, the present analysis combined with recent conformal bootstrap results, might be useful in order to get more insight in higher-dimensional CFTs outside the critical point.

The present method can be also applied in cases where the critical model is perturbed with more than one relevant operator. ${ }^{5}$ As an example, it would be interesting to apply it to the Ising model perturbed with both the magnetic field and reduced temperature in order to analyze the interplay of scales due to the coexistence of two kinds of perturbations. Finally, results from Conformal Perturbation Theory (CPT) can be useful in connection with sum rules as in the QCD case [43] or in the quantum critical case [33,44].

\footnotetext{
${ }^{4}$ Another interesting application of the present method is the analysis of the transport properties near the critical point in the presence of spontaneous symmetry breaking of translations. In this case, the results can be compared with the analogous holographic computation (see e.g. Refs. [35-40]).

${ }^{5}$ See e.g. Refs. [41,42] for other methods to treat CFTs perturbed by relevant operators.
}

\section{ACKNOWLEDGMENTS}

Special thanks goes to Michele Caselle, Slava Rychkov, and William Witczak-Krempa for providing comments on a preliminary version of the present paper. We would like also to thank Daniele Musso for useful conversations. Nicodemo Magnoli is thankful for the support of Instituto Nazionale di Fisica Nucleare (INFN) Scientific Initiative SFT: Statistical Field Theory, Low-Dimensional Systems, Integrable Models and Applications.

\section{APPENDIX: MELLIN TRANSFORM AND ASYMPTOTIC PROPERTIES OF INTEGRALS}

The structure of the integrals that we have to calculate is of the following form,

$$
I(m)=\int d^{2} z \Theta(m|z|) g(z),
$$

where $\Theta(m|z|)=e^{-m|z|}$ is an infrared cutoff function which can be eventually set to 0 at the end of the calculation. In order to obtain the asymptotic $(m \sim 0)$ expansion of the integral, we will make use of the Mellin transform of $I(m)$. Assuming that the leading behaviors of $I(m)$ are $m^{a}$ when $m \rightarrow 0$ and $m^{-b}$ when $m \rightarrow \infty$, one can define the Mellin transform $\tilde{I}(s)$ on the strip $-a<\operatorname{Re}(s)<b$ in the complex $s$ plane as

$$
\tilde{I}(s)=\int_{0}^{\infty} \frac{d m}{m} m^{s} I(m) .
$$

It is well known, see Ref. [45], that the poles of the Mellin transform are in one-to-one correspondence with the asymptotic expansion of the original function at $m=0$. In fact, one can write

$$
I(m)=\left.\sum_{i} \operatorname{Res}(\tilde{I}(s)) m^{-s}\right|_{s=-a_{i}},
$$

where $a_{1} \equiv a<a_{2}<\ldots$ are the powers of $m$ in the asymptotic expansion of $I(m)$ at $m \sim 0$. This equation tells us that, if the infrared counterterms do not give any finite contribution, then we can get the corrections to the Wilson coefficients by taking the residue of the perturbative expansions at $s=0$.

The Mellin transform previously described is particularly useful for our purposes if one notes that, by making use of the convolution theorem, it is possible to express the integral (A1) as

$$
\tilde{I}(s)=\Gamma(s) \tilde{g}(1-s)
$$

where

$$
\tilde{g}(1-s)=\int d^{2} z|z|^{-s} g(z)
$$


is essentially the Mellin transform of $g$ up to angular coefficients. Eventually, if one knows how to compute the Mellin transform of $g$, the integrals necessary to evaluate the derivative corrections of the Wilson coefficients are easily done. In our case, all the integrals have the following form:

$$
I(m ; x)=\int d^{2} z e^{-m|z|}|z|^{2 \alpha}|z-x|^{2 \gamma}|z-1|^{2 \beta} .
$$

The Mellin transform of this integral has been computed in Ref. [46] and is given by

$$
\tilde{I}(s ; x)=\Gamma(s) D(\alpha-s / 2, \beta, \gamma, x),
$$

where the integral

$$
D(a, b, c, x)=\int d^{2}|z|^{2 a}|z-x|^{2 c}|z-1|^{2 b}
$$

can be expressed as

$D(a, b, c, x)=\frac{S(a) S(c)}{S(a+c)}\left|I_{0 x}\right|^{2}+\frac{S(b) S(a+b+c)}{S(a+c)}\left|I_{1 \infty}\right|^{2}$.

In the last expression, $S(a) \equiv \sin (\pi a)$, and

$$
\begin{gathered}
I_{0 x} \equiv x^{1+a+c} \frac{\Gamma(a+1) \Gamma(c+1)}{\Gamma(a+c+2)} \\
\quad \times{ }_{2} F_{1}(-b, a+1, a+c+2, x), \\
I_{1 \infty} \equiv \frac{\Gamma(-a-b-c-1) \Gamma(b+1)}{\Gamma(-a-c)} \\
\times{ }_{2} F_{1}(-a-b-c-1,-c,-a-c, x),
\end{gathered}
$$

where ${ }_{2} F_{1}$ is the hypergeometric Gauss function.

\section{Integrals for the trapped Ising model}

In this subsection, we sketch the form of the integrals relevant to compute the perturbations of the trapped Ising model analyzed in Sec. IIIB. These integrals can be evaluated by using the Mellin transform technique previously described. In this respect, it is sufficient to consider just the first terms in the integrals (3.23)-(3.26). Eventually, considering a generic value of $p$, and after evaluating the $\left|z_{3}\right| \rightarrow 0$ limit, one has to compute the following integrals:

$\partial_{\rho} C_{\mathcal{E} \mathcal{E}}^{\sigma}\left(z_{1}\right)=-\left|z_{1}\right|^{p} \int d^{2} w|w|^{p-1}\left|w-\frac{1}{2}\right|^{2}|w-1|^{-1}$,

$$
\partial_{\rho} C_{\sigma \mathcal{E}}^{\mathbb{1}}\left(z_{1}\right)=-\frac{\left|z_{1}\right|^{p+\frac{3}{4}}}{2} \int d^{2} w|w|^{p-1}|w-1|^{\frac{3}{4}}
$$

$\partial_{\rho} C_{\sigma \mathcal{E}}^{\mathcal{E}}\left(z_{1}\right)=-\frac{\left|z_{1}\right|^{p+\frac{7}{4}}}{4} \int d^{2} w|w|^{p-1}|w-1|^{-\frac{1}{4}}|w+1|^{2}$,

$$
\begin{aligned}
\partial_{\rho} C_{\sigma \sigma}^{\sigma}\left(z_{1}\right)= & -2\left|z_{1}\right|^{p+\frac{7}{4}} \int d^{2} w|w|^{\frac{3}{2}}|w-1|^{-(p-s+4)} \mid w \\
& +\left.1\right|^{-(p-s+3)} .
\end{aligned}
$$

As one can easily see, the previous integrals are of the form (A6) and can be evaluated by using the Mellin technique transform described previously.
[1] S. El-Showk, M. F. Paulos, D. Poland, S. Rychkov, D. Simmons-Duffin, and A. Vichi, Solving the 3D Ising model with the conformal bootstrap, Phys. Rev. D 86, 025022 (2012).

[2] S. El-Showk, M. F. Paulos, D. Poland, S. Rychkov, D. Simmons-Duffin, and A. Vichi, Solving the 3d Ising model with the conformal bootstrap II. c-minimization and precise critical exponents, J. Stat. Phys. 157, 869 (2014).

[3] F. Gliozzi, More Constraining Conformal Bootstrap, Phys. Rev. Lett. 111, 161602 (2013).

[4] F. Gliozzi and A. Rago, Critical exponents of the 3d Ising and related models from conformal bootstrap, J. High Energy Phys., 10, 042 (2014).
[5] F. Kos, D. Poland, D. Simmons-Duffin, and A. Vichi, Bootstrapping the $\mathrm{O}(\mathrm{N})$ Archipelago, J. High Energy Phys. 11 (2015) 106.

[6] A. B. Zamolodchikov, Integrable field theory from conformal field theory, Adv. Stud. Pure Math. 19, 641 (1989).

[7] A. B. Zamolodchikov, Renormalization group and perturbation theory near fixed points in two-dimensional field theory, Yad. Fiz. 46, 1819 (1987) [Sov. J. Nucl. Phys. 46, 1090 (1987)].

[8] M. Gell-Mann and F. Low, Bound states in quantum field theory, Phys. Rev. 84, 350 (1951).

[9] K. G. Wilson, Nonlagrangian models of current algebra, Phys. Rev. 179, 1499 (1969). 
[10] R. Guida and N. Magnoli, All order IR finite expansion for short distance behavior of massless theories perturbed by a relevant operator, Nucl. Phys. B471, 361 (1996).

[11] M. Caselle, P. Grinza, and N. Magnoli, Short distance behavior of correlators in the 2-D Ising model in a magnetic field, Nucl. Phys. B579, 635 (2000).

[12] M. Caselle, P. Grinza, and N. Magnoli, Correction induced by irrelevant operators in the correlators of the 2-d Ising model in a magnetic field, J. Phys. A 34, 8733 (2001).

[13] M. Caselle, P. Grinza, R. Guida, and N. Magnoli, A new class of short distance universal amplitude ratios, J. Phys. A 37, L47 (2004).

[14] A. B. Zamolodchikov, Two point correlation function in scaling Lee-Yang model, Nucl. Phys. B348, 619 (1991).

[15] R. Hecht, Correlation functions for the two-dimensional ising model, Phys. Rev. 158, 557 (1967).

[16] E. A. Cornell and C. E. Wieman, Nobel lecture: Boseeinstein condensation in a dilute gas, the first 70 years and some recent experiments, Rev. Mod. Phys. 74, 875 (2002).

[17] W. Ketterle, Nobel lecture: When atoms behave as waves: Bose-einstein condensation and the atom laser, Rev. Mod. Phys. 74, 1131 (2002).

[18] T. Donner, S. Ritter, T. Bourdel, A. Öttl, M. Köhl, and T. Esslinger, Critical behavior of a trapped interacting bose gas, Science 315, 1556 (2007).

[19] I. Bloch, J. Dalibard, and W. Zwerger, Many-body physics with ultracold gases, Rev. Mod. Phys. 80, 885 (2008).

[20] D. Pappadopulo, S. Rychkov, J. Espin, and R. Rattazzi, Operator product expansion convergence in conformal field theory, Phys. Rev. D 86, 105043 (2012).

[21] J. S. Schwinger, The theory of quantized fields. 1., Phys. Rev. 82, 914 (1951).

[22] J. S. Schwinger, The theory of quantized fields. 2., Phys. Rev. 91, 713 (1953).

[23] P. H. Ginsparg, in Applied Conformal Field Theory, Proceedings of the Les Houches Summer School, Session XX, Fields, Strings, Critical Phenomena edited by E. Brezin and J. Zinn-Justin (North-Holland, Amsterdam, 1990), p. 640.

[24] M. Campostrini and E. Vicari, Critical Behavior and Scaling in Trapped Systems, Phys. Rev. Lett. 102, 240601 (2009).

[25] G. Costagliola, Operator product expansion coefficients of the 3D Ising model with a trapping potential, Phys. Rev. D 93, 066008 (2016).

[26] I. Gel'fand and G. Shilov, Generalized Functions: Properties and Operations, translated by E. Saletan (Academic Press, New York, 1964).

[27] M. Caselle, G. Delfino, P. Grinza, O. Jahn, and N. Magnoli, Potts correlators and the static three-quark potential, J. Stat. Mech. (2006) P03008.

[28] R. Guida and N. Magnoli, Vacuum expectation values from a variational approach, Phys. Lett. B 411, 127 (1997).

[29] Z. Komargodski and D. Simmons-Duffin, The random-bond Ising model in 2.01 and 3 dimensions, J. Phys. A 50, 154001 (2017).
[30] M. Caselle, G. Costagliola, and N. Magnoli, Numerical determination of the operator-product-expansion coefficients in the 3D Ising model from off-critical correlators, Phys. Rev. D 91, 061901 (2015).

[31] M. Caselle, G. Costagliola, and N. Magnoli, Conformal perturbation of off-critical correlators in the 3D Ising universality class, Phys. Rev. D 94, 026005 (2016).

[32] F. Kos, D. Poland, D. Simmons-Duffin, and A. Vichi, Precision islands in the Ising and $\mathrm{O}(\mathrm{N})$ models, J. High Energy Phys. 08 (2016) 036.

[33] A. Lucas, S. Gazit, D. Podolsky, and W. Witczak-Krempa, Dynamical Response near Quantum Critical Points, Phys. Rev. Lett. 118, 056601 (2017).

[34] V. A. Novikov, M. A. Shifman, A. I. Vainshtein, and V. I. Zakharov, Wilson's operator expansion: Can it fail?, Yad. Fiz. 41, 1063 (1985) [Nucl. Phys. B249, 445 (1985)].

[35] A. Amoretti, A. Braggio, N. Maggiore, and N. Magnoli, Thermo-electric transport in gauge/gravity models, Adv. Phys. 2, 409 (2017).

[36] A. Amoretti, D. Aren, R. Argurio, D. Musso, and L. A. Pando Zayas, A holographic perspective on phonons and pseudo-phonons, J. High Energy Phys. 05 (2017) 051.

[37] A. Amoretti, M. Baggioli, N. Magnoli, and D. Musso, Chasing the cuprates with dilatonic dyons, J. High Energy Phys. 06 (2016) 113.

[38] A. Amoretti, Condensed Matter Applications of AdS/CFT: Focusing on Strange Metals, (Springer Thesis, Cham, 2017).

[39] A. Amoretti, G. Caruso, N. Maggiore, and N. Magnoli, Holography in flat spacetime: 4D theories and electromagnetic duality on, J. High Energy Phys. 04 (2017), 142.

[40] A. Amoretti, A. Blasi, N. Maggiore, and N. Magnoli, Three-dimensional dynamics of four-dimensional topological BF theory with boundary, New J. Phys. 14, 113014 (2012).

[41] C. Behan, L. Rastelli, S. Rychkov, and B. Zan, Long-range critical exponents near the short-range crossover, Phys. Rev. Lett. 118, 241601 (2017).

[42] C. Behan, L. Rastelli, S. Rychkov, and B. Zan, A scaling theory for the long-range to short-range crossover and an infrared duality, J. Phys. A 50, 354002 (2017).

[43] M. Shifman, Vacuum structure and QCD sum rules: Introduction, Int. J. Mod. Phys. A 25, 226 (2010).

[44] A. Lucas, T. Sierens, and W. Witczak-Krempa, Quantum critical response: From conformal perturbation theory to holography, 2017.

[45] R. Wong, Asymptotic Approximations of Integrals: 3. Mellin Transform Techniques, Classics in Applied Mathematics, Society for Industrial and Applied Mathematics, (2001) p. 147.

[46] Vl. S. Dotsenko, and A. M. Polyakov, Fermion representations for the $2 \mathrm{~d}$ and $3 \mathrm{~d}$ ising models, Adv. Stud. Pure Math. 16, 123 (1987); Conformal field theory and solvable lattice models, editor by M. Jimbo, T. Miwa, and A. Tsuchiya (Academic Press, Cambridge, Massachusetts, 1988), p. 426 ISBN 0-12-385340-0. 